\title{
Peritoneal Migration of an Abdominally Implanted Epicardial Pacemaker: A Cause of Intestinal Obstruction
}

\author{
CARLEN GOMEZ, MACDONALD DICK II, RAMIRO HERNANDEZ, \\ ARNOLD G. CORAN, DENNIS CROWLEY, and GERALD A. SERWER \\ From C.S. Mott Children's Hospital, University of Michigan, Ann Arbor, Michigan
}

GOMEZ, C., ET AL.: Peritoneal Migration of an Abdominally Implanted Epicardial Pacemaker: A Cause of Intestinal Obstruction. We report the case of a 10-year-old child with an abdominally implanted epicardial pacemaker that eroded through the peritoneum and migrated to an intraperitoneal location, resulting in partial and then complete intestinal obstruction. This potentially life-threatening complication should be considered when a patient with an abdominally implanted pacemaker presents with abdominal pain.

(PACE 1995; 18:2231-2232)

pacemaker, children, intestinal obstruction

\section{Introduction}

Over the past 2 decades the use of permanent cardiac pacemakers has increased in the young due to improvement in the diagnosis of cardiac arrhythmias in children, advances in neonatal and infant cardiovascular surgery, and miniaturization of generator microcircuitry and power source. Although transvenous pacing is more common in younger children than in the past, epicardial pacing is still used in children under $10 \mathrm{~kg}$ and in children with intracardiac shunts. ${ }^{1}$ Complications of epicardial pacemakers are related to lead problems such as high thresholds and early exit block, as well as premature battery depletion and pocket erosion and infection. ${ }^{2}$ We report a unique case of an abdominal epicardial pacemaker eroding through the peritoneum causing bowel obstruction 9 years after the pacemaker was implanted.

\section{Case Report}

A 10-year-old female received a pacemaker at 2 weeks of age because of congenital complete heart block and complex congenital heart disease,

Address for reprints: Carlen Gomez, M.D., Pediatric Cardiology, F1310 Box 0204, University of Michigan Medical Center, Ann Arbor, MI 48109-0204. Fax: (313) 936-9470.

Received June 19, 1995; revision July 31, 1995; accepted August $1,1995$. followed by a heart transplant at 7 months of age because of intractable heart failure. At 9 years of age, the patient complained of intermittent abdominal discomfort. At 10 years of age, she awoke from sleep complaining of abdominal pain and then vomited bile tinged material. The next day, she was hospitalized for dehydration and possible bowel obstruction. Pertinent physical findings included a temperature of $37.3 \mathrm{C}$, a soft, nondistended, mildly tender abdomen with faint bowel sounds and no hepatosplenomegaly. Relevant laboratory data included a white blood cell count of 24,600 cells/cc, blood urea nitrogen concentration $34 \mathrm{mg} / \mathrm{dL}$, and creatinine concentration $1.3 \mathrm{mg} /$ dL. Chest X ray was unchanged. Abdominal films revealed dilated loops of small bowel, air fluid levels (upright film), and little gas from colon to rectum. The pacemaker was in the right lower quadrant, unchanged from previous films. An abdominal computed assisted tomographic scan (CT) demonstrated mildly dilated fluid-filled small bowel loops throughout the abdomen. The pacemaker was located within the peritoneum in the pelvis (Fig. 1).

The patient was taken to the operating room for probable complete bowel obstruction. The pacemaker was found within the peritoneal cavity in the right lower quadrant. Adhesive bands crossed the abdominal cavity from the pacemaker and inserted on the left ovary and fallopian tube resulting in complete small bowel obstruction. 


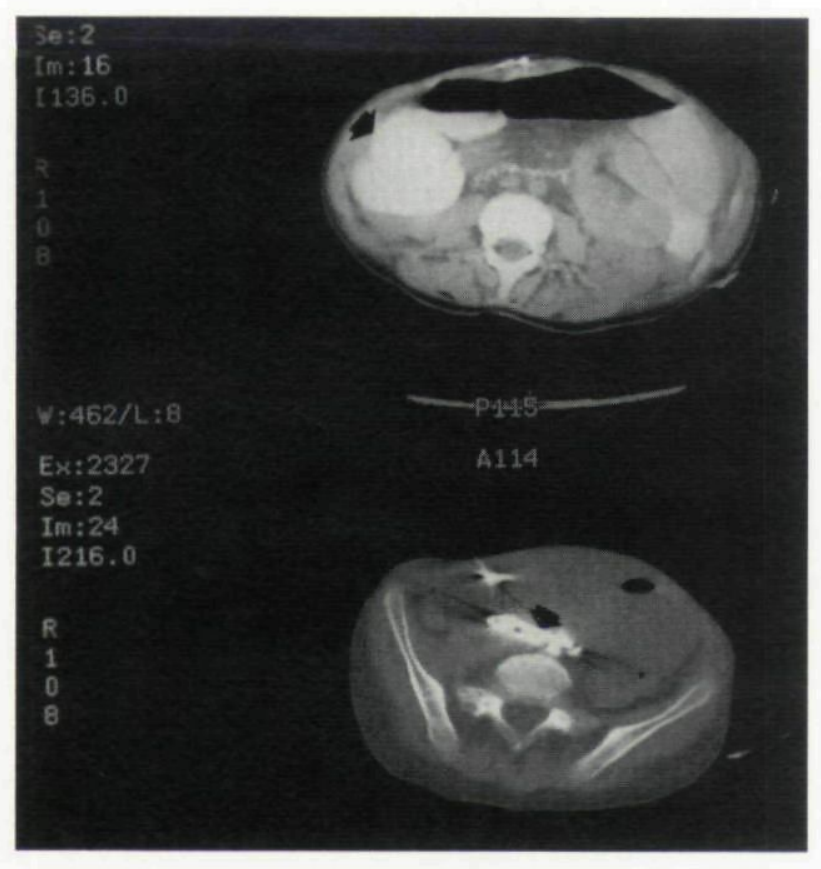

Figure 1. Cross-sectional CT scan of abdomen (top image) shows dilated loops (arrow) of fluid filled bowel with normal caliber ascending and descending colon. The pacemaker (lower image, arrow) is seen in peritoneal cavity just anterior to the psoas muscle at the level of $L 5$.

When the adhesions were divided, the small bowel obstruction was relieved. No necrosis of the bowel was noted. The pacemaker and intraabdominal segments of the leads were removed. The patient had an uneventful postoperative course and returned to her usual state of health.

\section{References}

1. Smith RT Jr. Pacemakers for children. In PC Gillette, A Garson Jr (eds.): Pediatric Arrhythmias: Electrophysiology and Pacing. New York, NY, Grune \& Stratton, 1990, p. 532.

2. Kerstjens-Frederikse MWS, Bink-Boelkens MTE, Jongste MJL, et al. Permanent cardiac pacing in children: Morbidity and efficacy of follow-up. Int J Cardiol 1991; 33:207-214.

3. Ott DA. Epicardial pacemaker implantation. In PC
The experience with this patient underscores a potentially life-threatening complication of abdominally implanted epicardial pacemakers. In small children, such as our patient, wound closure of the abdominal pacemaker pocket with minimal tension on the suture line, as well as limited pacemaker movement, is facilitated by the creation of a pocket deep to the rectus abdominis muscle and superficial to the posterior rectus fascia. ${ }^{3}$ Van Hare et al. ${ }^{4}$ reported a patient with an abdominally implanted pacemaker that migrated into the pericardial space 4 months after implantation; he postulated that the generator tracked along the lead path into the pericardial sac. In contrast, the pacemaker in our patient eroded away from the lead track and into the peritoneal cavity over a period of 9 years.

The number of children with pacemakers is increasing due to heightened awareness of cardiac arrhythmias and longer survival of young patients with complex congenital heart disease. ${ }^{5}$ Improved technology and techniques produce longer functioning pacing systems that may require less frequent revision, allowing for possible migration of the generator to adjacent locations. However, in view of the rare complications of the submuscular approach, ${ }^{3}$ its advantages for epicardial pacemakers in infants and small children outweigh the very small risk of generator erosion and migration into the peritoneal cavity. Nonetheless, chronic and/or intermittent abdominal pain in a patient with an abdominally implanted pacemaker should prompt investigation of the pacemaker's precise location within the abdomen, possibly necessitating a CT scan.

Gillette, A Garson Jr (eds.): Pediatric Arrhythmias: Electrophysiology and Pacing. New York, NY, Grune \& Stratton, 1990, pp. 575-579.

4. Van Hare GF, Witherell C, Merrick SM. Migration of an epicardial pacemaker to the pericardial space in an infant. PACE 1994; 17:1808-1810.

5. Gillette PC, Zeigler VL, Winslow AT, et al. Cardiac pacing in neonates, infants, and preschool children. PACE 1992; 15:2046-2049. 
This document is a scanned copy of a printed document. No warranty is given about the accuracy of the copy. Users should refer to the original published version of the material. 